\title{
Active Microwave Imaging of Inhomogeneous Bodies
}

\author{
CHRISTIAN PICHOT, LUIS JOFRE, GUILLAUME PERONNET, AND JEAN-CHARLES BOLOMEY
}

\begin{abstract}
A numerical method and experimental technique for microwave imaging of inhomogenous bodies is presented. This method is based on the interpretation of the diffraction phenomena and leads to tomographic reconstruction of the body under investigation. Various numerical examples are given on spatial impulse response, recognition of dielectric rods, inhomogeneous bodies, and simulated human arm. Different experimental results on dielectric rods and isolated animal organs are also given.
\end{abstract}

\section{INTRODUCTION}

$\mathbf{D}_{\mathrm{a}}$ URING THE LAST few years, it has been possible to observe fantastic development of tomographic processes for biomedical purposes. X-rays, ultrasonic waves and, more recently, nuclear magnetic resonance are the most popular examples.

Oddly, active imaging systems based on microwaves are in the beginning phase [1]-[7], [9], [11]-[13], [15]-[17], [28], [29]. However, there are some reasons for that. A priori ideas are the difficult penetration of microwaves in living tissues, their high attenuation constant, and their poor resolution.

In fact, the first experiments of Larsen and Jacobi [1]-[3] revealed that these drawbacks can be overcome and that microwave imaging had some potentialities, mainly resulting from the specificity of the interaction mechanisms with the living tissues, different from those encountered with X-rays or ultrasonic waves for instance.

The problem of field penetration into high water content tissues can be solved by a careful matching of the applicators; the attenuation rate is compatible with the present state-of-the art in the receiver sensivity area for thicknesses as large as a few ten centimeters. ${ }^{1}$ Finally, the resolution is better than could be imagined because of the high permittivity constant of tissues with large water content. For example, the wavelength in such tissues is about one centimeter at an operating frequency equal to $3 \mathrm{GHz}$.

Thus, the expected resolution, on the Rayleigh criterion basis, is of the order of $6 \mathrm{~mm}$, i.e., about half a wavelength at $3 \mathrm{GHz}$ in water. This has been confirmed both on numerical simulations and experimentations. If X-rays CAT scanners have better resolution (fraction of $\mathrm{mm}$ ), the information available in a microwave image is potentially quite different.

The experimental processes depend on the reconstruction algorithm to be used. The first method, strongly related to $\mathrm{X}$-ray tomodensitometry, is based on a linear path assumption between an emitter and a receiver. Measuring the transmission coefficient during the simultaneous displacement of the

Manuscript received September 16, 1983; revised November 19, 1984. This work was supported by DGRST under Contract $81 \mathrm{M} 0909$ to $81 \mathrm{M} 0911$ and by MRI under Contract $82 \mathrm{M} 1485$.

C. Pichot, G. Peronnet, and J. C. Bolomey are with the Laboratoire des Signaux et Systémes, Groupe d'Electromagnétisme, Plateau du Moulon, 91190 Gif sur Yvette, France.

L. Jofre is with the Laboratoire des Signaux et Systemes, Group d'Electromagnétisme, Plateau du Moulon, 91190 Gif sur Yvette, France and with Escuela Técnica Superior de Ingenieros de Telecommunicación, c/Jordi Girona Salgado, s/n, Barcelona, Spain.

1 Taking into account the limitation of the incident power density for a total harmlessness under the safety standard $\left(<10 \mathrm{~mW}^{\circ} \mathrm{cm}^{2}\right)$. antennas provides one projection. From many projections corresponding to different orientations, it is possible to deduce cross sections of the volume under test. The second method belongs to holographic techniques. For a given irradiation of this volume, the scattered field is recorded on a finite surface (hologram) and the image of the domain under investigation is numerically obtained by simulating the effect of an optical focusing system. Such an approach is based on a better description of the microwave-tissues interaction. However, comparisons are not yet available to enable conclusions with respect to the advantages of each method.

This paper is devoted to the description of efficient algorithms for the diffraction tomography. By efficient, one means that the required computations can be done in quasireal time in realistic situations. These situations resulted from experiments conducted on phantoms or perfused organs, and from "exact" numerical simulations. These experiments used a fast microwave camera, which has been described elsewhere [13], [15], providing a recording duration of the order of the reconstruction time. Besides, numerical simulation was found to be very useful for fine estimation of contrasts and complementary to the experimental approach.

In this paper, first the tomographic algorithm is presented for two-dimensional inhomogeneities. Second numerical results obtained from simulation are given in order to evaluate the spatial impulse response, the resolution, and the accessible contrast. Then the reconstruction of inhomogeneous and complex bodies is considered.

The third section is devoted to the description of the experimental setup and to the discussion of the result obtained on dielectric rods and isolated organ (perfused and nonperfused horse kidney).

\section{TWO-DIMENSIONAL THEORY}

One considers a cylindrical object of arbitrary cross section $S$ which is characterized by a dielectric permittivity $\epsilon_{S}(x, y)$ and a conductivity $\sigma_{S}(x, y)$. This object is illuminated by a plane wave for which the electric field $\vec{E}^{i}$ is parallel to the cylinder axis ( $z$ axis) as depicted in Fig. 1(a).

The external medium is assumed to be uniform and characterized by a dielectric permittivity $\epsilon$ and conductivity $\sigma$. The permeability is that of vacuum $\mu=\mu_{0}$ in each medium. Let $\vec{E}$ represents the total electric field and $\vec{E}^{d}$ the diffracted field which is generated by the equivalent electric current radiating in the external medium so that

$$
\vec{E}=\vec{E}^{i}+\vec{E}^{d}
$$

where the equivalent electric currents are defined as usually

$$
\vec{J}_{s}(x, y)=\left(k_{s}^{2}(x, y)-k^{2}\right) \vec{E}(x, y)
$$

with

$$
k_{s}(x, y)=\sqrt{\omega^{2} \mu_{0} \epsilon_{s}(x, y)-i \omega \mu_{0} \sigma_{s}(x, y)}
$$




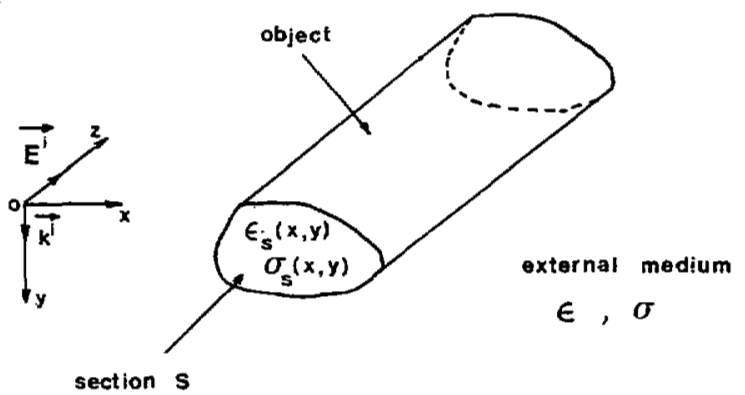

(a)

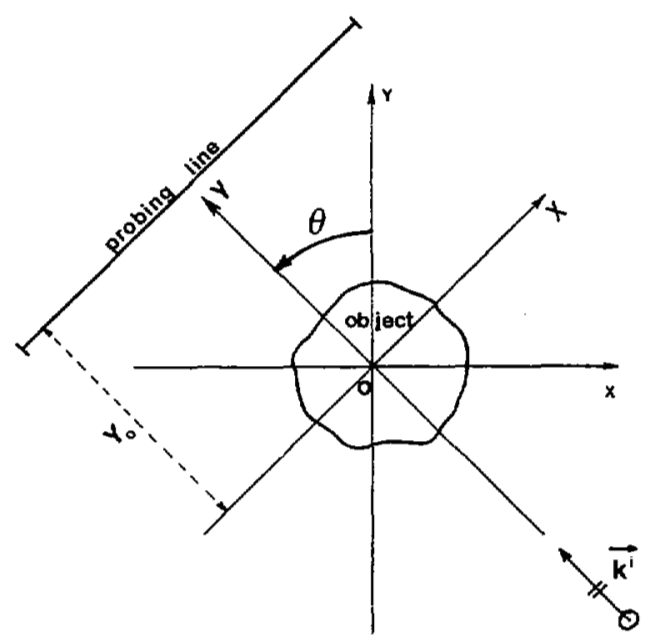

(b)

Fig. 1. (a) Two-dimensional inhomogeneous object and coordinate system. (b) Cross section of the object and data acquisition.

$$
k=\sqrt{\omega^{2} \mu_{0} \epsilon-i \omega \mu_{0} \sigma}
$$

for a time dependence $e^{i \omega t}$.

The total field $\vec{E}$ can be exactly calculated with an integral representation (Richmond [19]):

$$
\vec{E}(x, y)=\vec{E}^{i}(x, y)+\iint_{S} \vec{J}_{s}\left(x^{\prime}, y^{\prime}\right) G\left(x, y ; x^{\prime}, y^{\prime}\right) d x^{\prime} d y^{\prime}
$$

for any point $(x, y)$ inside or outside the object. The Green's function $G\left(x, y, x^{\prime}, y^{\prime}\right)$ is given as (Tyras [23] for the integral representation)

$$
\begin{aligned}
& G\left(x, y ; x^{\prime}, y^{\prime}\right) \\
& =-\frac{i}{4} H_{0}^{(2)}\left(k \sqrt{\left(x-x^{\prime}\right)^{2}+\left(y-y^{\prime}\right)^{2}}\right) \\
& =-\frac{i}{2} \int_{-\infty}^{+\infty} \frac{e^{-i \sqrt{k^{2}-4 \pi^{2} \nu^{2}\left|y-y^{\prime}\right|}}}{\sqrt{k^{2}-4 \pi^{2} \nu^{2}}} e^{-i 2 \pi \nu\left(x-x^{\prime}\right)} d \nu
\end{aligned}
$$

with

$$
\operatorname{Im}\left(\sqrt{k^{2}-4 \pi^{2} \nu^{2}}\right) \leqslant 0 .
$$

One notes that the $\vec{E}^{i}, \vec{E}$, and $\vec{E}^{d}$ vectors have only $z$ component.

The solution of the direct problem (computation of the total or diffracted field from the knowledge of the geometrical and electromagnetic characteristics of the object with a given incident field) can be carried out with the moment method (Richmond [19], Iskander et al. [24], [25]) or in some cases with an itera- tive technique in connection with the Born approximation (Barthes and Vasseur [22]). For the inverse problem which consists of finding the shape and electromagnetic characteristics $\epsilon_{S}(x, y)$ and $\sigma_{S}(x, y)$ of the object for a given incident field, when the diffracted field is measured along a segment line, equivalent methods can be applied (Barthes and Vasseur [22], Ghodgaonkar et al. [26]). However, powerful computers are needed when the cross-section dimensions of the bodies are of the order of one wavelength or more.

The following method for the inverse problem can readily be used on a minicomputer or array processor.

Consider the case where a plane wave is incident at an angle $\theta$ with respect to $y$ axis and where the probing line for diffracted field data is perpendicular to the incident propagation vector $\vec{k}^{i}$ (see Fig. 1(b)). Let $\psi(\theta ; x, y)$ represent the normalized diffracted field as

$$
\psi(\theta ; x, y)=\frac{E_{z}^{d}(\theta ; x, y)}{E_{z}^{i}(\theta ; x, y)}
$$

where $E_{z}^{d}(\theta ; x, y)$ is the diffracted field related to the incident field $E_{z}^{i}(\theta ; x, y)\left(E_{z}^{i}(\theta ; x, y)=e^{-i k(-x \sin \theta+y \cos \theta)}\right)$. Introducing $\psi(\theta ; x, y)$ in (4) leads to

$$
\begin{aligned}
& \psi(\theta ; x, y) \\
& =\left[E_{z}^{i}(\theta ; x, y)\right] \iint_{S\left(x^{\prime}, y^{\prime}\right)} E_{z}^{i}\left(\theta ; x^{\prime}, y^{\prime}\right) K\left(\theta ; x^{\prime}, y^{\prime}\right) \\
& \cdot G\left(x, y ; x^{\prime}, y^{\prime}\right) d x^{\prime} d y^{\prime}
\end{aligned}
$$

where

$$
K(\theta ; x, y)=\left(k_{s}^{2}(x, y)-k^{2}\right)(1+\psi(\theta ; x, y))
$$

is the normalized electric current. Let us define the new frame $(X, Y)$ as

$$
\begin{array}{ll}
X=x \cos \theta+y \sin \theta & x=X \cos \theta-Y \sin \theta \\
Y=-x \sin \theta+y \cos \theta & y=X \sin \theta+Y \cos \theta
\end{array}
$$

Equation (7) becomes

$$
\begin{aligned}
& \Psi(\theta ; X, Y) \\
& =e^{i k Y} e^{-i k Y} \iint_{S\left(X^{\prime}, Y^{\prime}\right)} E_{z}^{i}\left(\theta ; X^{\prime}, Y^{\prime}\right) K\left(\theta ; X^{\prime} \cos \theta\right. \\
& \left.\quad-Y^{\prime} \sin \theta, X^{\prime} \sin \theta+Y^{\prime} \cos \theta\right) \\
& \quad \cdot G\left(X, Y ; X^{\prime}, Y^{\prime}\right) d X^{\prime} d Y^{\prime}
\end{aligned}
$$

with $\Psi(\theta, X, Y)$ normalized diffracted field in the new frame. The use of the integral representation (5) of $G\left(X, Y ; X^{\prime}, Y^{\prime}\right)$ yields

$$
\Psi(\theta ; X, Y)
$$

$$
\begin{aligned}
= & -\frac{i}{2} e^{i k Y} \int_{-\infty}^{+\infty} \iint_{S\left(X^{\prime}, Y^{\prime}\right)} e^{-i k Y^{\prime}} K\left(\theta ; X^{\prime} \cos \theta\right. \\
& \left.-Y^{\prime} \sin \theta, X^{\prime} \sin \theta+Y^{\prime} \cos \theta\right) \frac{e^{-i \sqrt{k^{2}-4 \pi^{2} \nu^{2}}\left|Y-Y^{\prime}\right|}}{\sqrt{k^{2}-4 \pi^{2} \nu^{2}}} \\
& \cdot e^{-i 2 \pi \nu\left(X-X^{\prime}\right)} d X^{\prime} d Y^{\prime} d \nu .
\end{aligned}
$$

Let $\hat{\Psi}(\theta ; \nu, Y)$ be the Fourier transform of $\Psi(\theta, X, Y)$ with re- 
spect to $X$

$$
\dot{\Psi}(\theta ; \nu, Y)=\int_{-\infty}^{+\infty} \Psi(\theta ; X, Y) e^{i 2 \pi \nu X} d X
$$

then from (10) we obtain

$\hat{\Psi}(\theta ; \nu, Y)$

$$
\begin{aligned}
= & -\frac{i}{2} e^{i k Y} \iint_{S\left(X^{\prime}, Y^{\prime}\right)} e^{-i k Y^{\prime}} K\left(\theta ; X^{\prime} \cos \theta\right. \\
& \left.-Y^{\prime} \sin \theta, X^{\prime} \sin \theta+Y^{\prime} \cos \theta\right) \frac{e^{-i \sqrt{k^{2}-4 \pi^{2} \nu^{2}}\left|Y-Y^{\prime}\right|}}{\sqrt{k^{2}-4 \pi^{2} \nu^{2}}} \\
& \cdot e^{i 2 \pi \nu X^{\prime}} d X^{\prime} d Y^{\prime} .
\end{aligned}
$$

For any $Y_{0}>\max \left\{Y^{\prime} \in S\left(X^{\prime}, Y^{\prime}\right)\right\}$ in the frame $(X, Y),(12)$ becomes

$\hat{\psi}\left(\theta ; \nu, Y_{0}\right)$

$=-\frac{i}{2} e^{i k Y_{0}} \frac{e^{-i \sqrt{k^{2}-4 \pi^{2} \nu^{2}} Y_{0}}}{\sqrt{k^{2}-4 \pi \tau^{2} \nu^{2}}} \iint_{S\left(X^{\prime}, Y^{\prime}\right)} K\left(\theta ; X^{\prime} \cos \theta\right.$

$$
\begin{aligned}
& \left.-Y^{\prime} \sin \theta, X^{\prime} \sin \theta+Y^{\prime} \cos \theta\right) \\
& \cdot e^{i 2 \pi\left(-k / 2 \pi+1 / 2 \pi \sqrt{k^{2}-4 \pi^{2} \nu^{2}}\right) Y^{\prime}} e^{i 2 \pi \nu X^{\prime}} d X^{\prime} d Y^{\prime}
\end{aligned}
$$

where

$$
\begin{gathered}
\iint_{S\left(X^{\prime}, Y^{\prime}\right)} K\left(\theta ; X^{\prime} \cos \theta-Y^{\prime} \sin \theta, X^{\prime} \sin \theta+Y^{\prime} \cos \theta\right) \\
\cdot e^{i 2 \pi\left(-k / 2 \pi+1 / 2 \pi \sqrt{\left.k^{2}-4 \pi^{2} \nu^{2}\right)} e^{i 2 \pi \nu X^{\prime}} d X^{\prime} d Y^{\prime}\right.} \\
=\iint_{S S\left(x^{\prime}, y^{\prime}\right)} K\left(\theta ; x^{\prime}, y^{\prime}\right) e^{i 2 \pi(\mu(\nu) \cos \theta+\nu \sin \theta) y^{\prime}} \\
\cdot e^{i 2 \pi(-\mu(\nu) \sin \theta+\nu \cos \theta) x^{\prime}} d x^{\prime} d y^{\prime}
\end{gathered}
$$

with

$$
\mu(\nu)=-\frac{k}{2 \pi}+\frac{1}{2 \pi} \sqrt{k^{2}-4 \pi^{2} \nu^{2}} .
$$

Let $\hat{K}(\theta ; \nu, \mu)$ be the two-dimensional Fourier transform of $K(\theta ; x, y)$

$$
\hat{\mathrm{K}}(\theta ; \nu, \mu)=\int_{-\infty}^{+\infty} \int_{-\infty}^{+\infty} K(\theta ; x, y) e^{i 2 \pi(\nu x+\mu y)} d x d y
$$

The relation (14) defines a usual two-dimensional Fourier transform of $K(\theta ; x, y)$ (its support is bounded to $S(x, y)$ so the integrals can be extended to infinity) on a curve $(\alpha(\theta ; \nu)$, $\beta(\theta ; \nu)$ ) only if we restrict $\mu(\nu)$ to $\Omega(\nu)=\operatorname{Real}(\mu(\nu))$.

In this case and from (13) we deduce a direct relation between $\hat{K}\left(\theta ; \alpha(\theta ; \nu), \beta(\theta ; \nu)\right.$ and $\hat{\Psi}\left(\theta ; \nu, Y_{0}\right)$ :

$$
\begin{aligned}
& \hat{K}(\theta ; \alpha(\theta ; \nu), \beta(\theta ; \nu)) \\
& \quad=2 i \sqrt{k^{2}-4 \pi^{2} \nu^{2}} e^{i 2 \pi \Omega(\nu) Y_{0}} \hat{\Psi}\left(\theta ; \nu, Y_{0}\right)
\end{aligned}
$$

with

$$
\alpha(\theta ; \nu)=\nu \cos \theta-\Omega(\nu) \sin \theta
$$

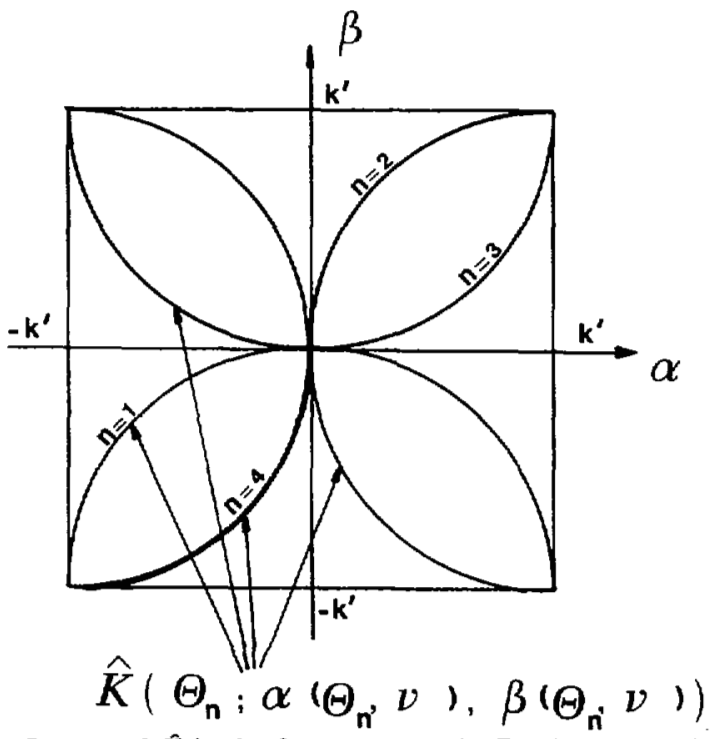

Fig. 2. Support of $\hat{K}$ in the frequency domain $D$ using four orthogonal rotations.

$$
\begin{aligned}
\beta(\theta ; \nu) & =\nu \sin \theta+\Omega(\nu) \cos \theta \\
\Omega(\nu) & =\operatorname{Re}(\mu(\nu)) .
\end{aligned}
$$

A choice on $k$ and $\nu$ yielding $\Omega(v)$ is to take $k^{\prime}=\operatorname{Re}(k)$ (instead of $k$ ) and $\nu<k^{\prime} / 2 \pi$. This choice leads to reconstruct in a lossless outer medium (of wavenumber $k^{\prime}$ ) and neglect the spatial frequency $\nu$ for $\nu>k^{\prime} / 2 \pi$. In order to combine every rotation information, let $\hat{P}(\theta ; \alpha, \beta)$ be the function defined in the spectrum domain as

$$
\hat{P}(\theta ; \alpha, \beta)= \begin{cases}1, & \text { if }(\nu, \mu) \epsilon(\alpha(\theta ; \nu), \beta(\theta ; \nu)) \\ 0, & \text { elsewhere. }\end{cases}
$$

For $N$ rotations $\theta_{n}\left(n=1, \cdots, N\right.$ with $\theta_{n}=2 \pi / N(n=1)$ and using the information obtained from each one, one reconstructs

$$
\hat{\tilde{K}}(\alpha, \beta)=\sum_{i=1}^{N} \hat{K}\left(\theta_{i} ; \alpha, \beta\right) \hat{P}\left(\theta_{i} ; \alpha, \beta\right)
$$

in the $D$ spectrum domain $\left(\alpha, \beta \in\left[-k^{\prime}, k^{\prime}\right]\right.$ ) (See Fig. 2 for $N=4$ ). One has a set of semicircles passing through the central point $(0,0)$. The function $\hat{\hat{K}}(\alpha, \beta)$ is the Fourier transform of the image $\widetilde{K}(x, y)$ :

$$
\tilde{K}(x, y)=\sum_{i=1}^{N}\left[\left(k_{s}^{2}(x, y)-k^{2}\right)\left(1+\psi\left(\theta_{i} ; x, y\right)\right)\right] * P\left(\theta_{i} ; x, y\right)
$$

and the asterisk means the convolution product.

The main problem when combining the different $\hat{P}\left(\theta_{i} ; \alpha, \beta\right)$ is the multiple values of $\hat{K}\left(\theta_{i}, \alpha, \beta\right)$ at a same point. The adopted method is to take the average value.

Note that in the Born approximation $\left(\psi\left(\theta_{i} ; x, y\right) \ll 1\right)$, the reconstructed image is

$$
\tilde{K}(x, y)=\left(k_{s}^{2}(x, y)-k^{2}\right) * \bar{P}(x, y)
$$

where $\vec{P}(x, y)=\sum_{i=1}^{N} P\left(\theta_{i} ; x, y\right)$ is the spatial impulse response of the system.

An important aspect of the reconstruction method is the numerical estimate of the $\hat{K}$ normalized equivalent current $\tilde{K}(x, y)$ from the knowledge of $K(\alpha, \beta)$ with a limited number of rota- 
tions [27]. This computation being based on a discrete Fourier transform (fast Fourier transform for example). The values of $\hat{\tilde{K}}(\alpha, \beta)$ are required on a uniform discrete lattice. This implies a problem of interpolation or weighting.

Practically the diffracted field is measured on a segment line. Thus, to take into account the length $a$ of the segment line, we define $\hat{\Psi}_{a}\left(\theta ; p, Y_{0}\right)$ the measure at $Y=Y_{0}$ as

$$
\begin{aligned}
\hat{\Psi}_{a}\left(\theta ; \nu, Y_{0}\right) & =\int_{-a / 2}^{a / 2} \Psi\left(\theta ; X, Y_{0}\right) e^{i 2 \pi X} d X \\
& =\hat{\pi}_{a}(\nu) * \hat{\Psi}\left(\theta ; \nu, Y_{0}\right)
\end{aligned}
$$

where

$$
\hat{\pi}_{a}(\nu)=\frac{\sin (\pi v a)}{\pi \nu} .
$$

\section{NUMERICAL RESULTS}

The spatial impulse response, the resolution, the contrast, the reconstruction of dielectric rods and complex bodies are now investigated.

The computation of the normalized diffracted field $\psi\left(\theta ; x, y_{0}\right)$ from (4) is obtained by means of the moment method, which leads to a system of linear equations [19], [24], [25]. The size of this system is function of the number of cells in the object (the rods in this case) where the electric field is supposed to be constant. In most cases the size of the cells does not exceed $0.2 \lambda_{0} / \sqrt{\epsilon}_{r_{S}}$

\section{A. Spatial Impulse Response}

The use of reconstruction procedure implies to know the influence of:

- spectrum limitation between $-k^{\prime}$ and $k^{\prime}\left(k=k^{\prime}-i k^{\prime \prime}\right)$;

- reconstruction in a lossless outer medium;

- sampling interval of the measured field and probing line length;

- number of views, (or rotations).

The spatial impulse response can give a qualitative and quantitative characterization of the image. With this goal, we suppose that $K(x, y)=\delta\left(x-x_{p}, y-y_{p}\right)$ where $\delta\left(x-x_{p}, y-y_{p}\right)$ is the Dirac distribution at $\left(x_{p}, y_{p}\right)$ point and we try to recover it using one view.

One the one hand let $\delta_{x}$ by the transversal spatial impulse response along $x$-axis.

On the other hand, let $\delta_{y}$ be the longitudinal spatial impulse response along $y$-axis.

As an example, Fig. 3 shows the values of $\delta_{x}$ and $\delta_{y}$ for a Dirac distribution $\delta(x, y)$ located at $x_{p}=0$ and $y_{p}=-1.16 \mathrm{~cm}$ in a lossy medium (water: $\epsilon_{r}=74, \sigma=2.39 \mathrm{~S} / \mathrm{m}$ ) at $3.0 \mathrm{GHz}$ (the wavelength in this medium is $\lambda=\lambda_{0} / \sqrt{\epsilon_{r}}=1.16 \mathrm{~cm}$ )

We first discuss the influence of the length $a$ of the probing line. From the elementary source $\delta(x, y)$ located in $P$, the probing line is seen with a half-aperture angle $\theta_{0}$.

In Fig. 4 are represented the results on $\delta_{x}$ and $\delta_{y}$ for different aperture angles $\theta_{0}\left(30^{\circ}, 45^{\circ}, 60^{\circ}\right.$, and $\left.90^{\circ}\right)$ in water medium at $3.0 \mathrm{GHz}$. The point source is located at $x_{p}=0$ and $y_{p}=-3.5$ $\mathrm{cm}=-3 \lambda$ and the measurement line is situated at $y_{0}=0$.

The sampling interval $\Delta x$ of the measured field is given by virtue of Shannon theorem under the assumption that the spectrum
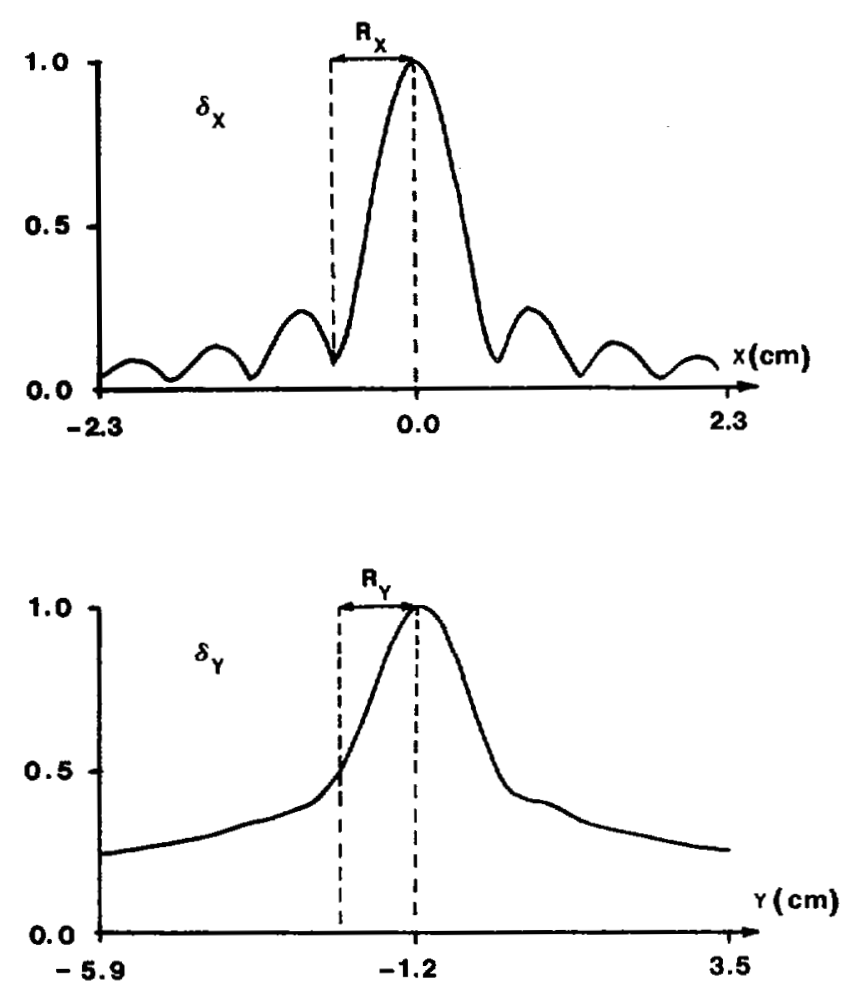

Fig. 3. Transversal $\delta_{x}$ and longitudinal $\delta_{y}$ spatial impulse response in water at $3 \mathrm{GHz} . R_{x}=\lambda / 2=0.58 \mathrm{~cm}, R_{y}=2 \lambda=2.32 \mathrm{~cm}$.
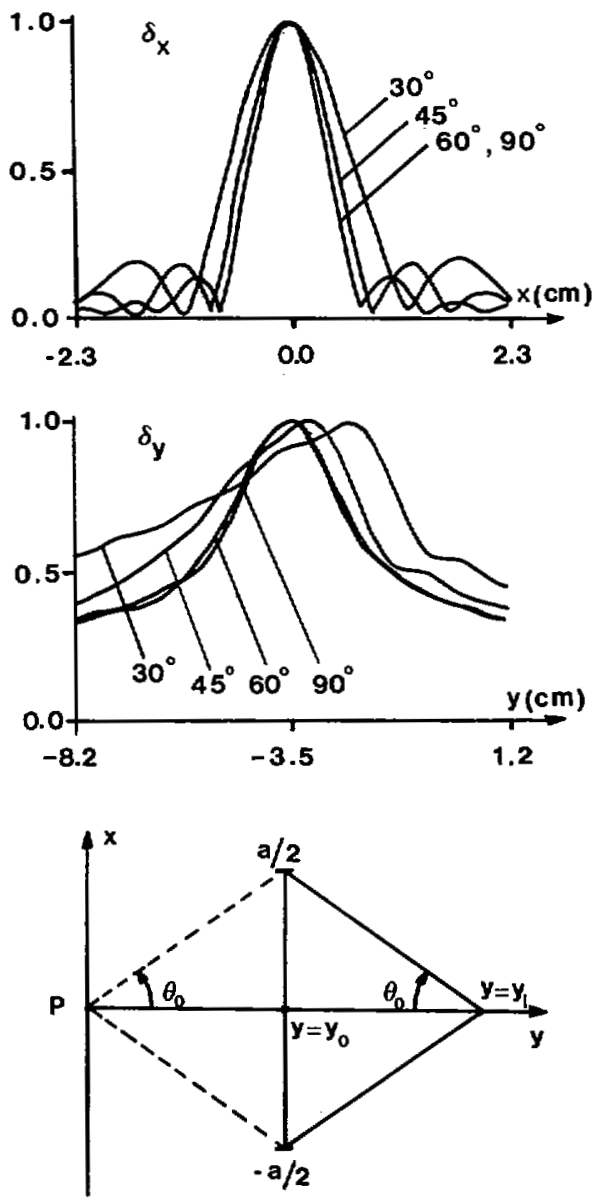

Fig. 4. Transveral $\delta_{x}$ and longitudinal $\hat{\delta}_{y}$ spatial impulse response for various aperture angles $\theta_{0}\left(\theta_{0}=30^{\circ}, 45^{\circ}, 60^{\circ}\right.$, and $\left.90^{\circ}\right)$. 


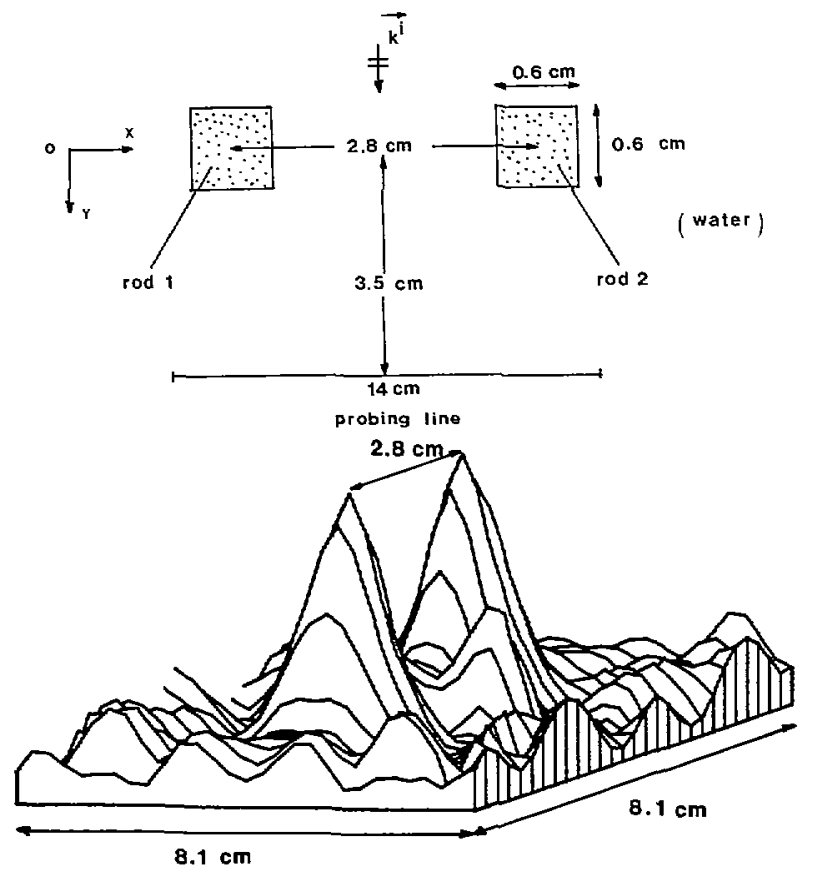

Fig. 5. Two identical rods $\left(\epsilon_{r_{1}}=\epsilon_{r 2}=2.4\right.$ and $\sigma_{1}=\sigma_{2}=0.0 \mathrm{~S} / \mathrm{m}$ ) separated by a distance of $2.8 \mathrm{~cm}$. The outer medium is water $\epsilon_{r}=74$ and $\sigma=2.39 \mathrm{~S} /$ m). Amplitude $|\tilde{K}(x, y)|$ of the reconstructed image.

is zero for $|\alpha|>\left|k^{\prime}\right|$ :

$$
\Delta x=\frac{\pi}{k^{\prime}}=\frac{\lambda}{2}
$$

(where $\lambda$ is the real part of the wavelength in the outer medium).

Concerning the spectrum truncature, the Fourier transform of the diffracted field is very attenuated for $\nu>k^{\prime} / 2 \pi$ at for a distance $y_{0}>\lambda / 2 \pi$.

So no significant information (in this medium) can be recovered for $y_{0}>\lambda / 2$, the spectrum being too much attenuated. However for $y_{0} \leqslant \lambda / 2$, the sampling internal can be taken $<\lambda / 2$ to take into account the evanescent waves.

Object rotation can improve significantly the spatial impulse response.

\section{B. Identical Rods}

The reconstruction of two lossless dielectric square rods surrounded by water is investigated at $3 \mathrm{GHz}$ frequency. The two rods are illuminated by a plane wave and their centers are separated by a distance of $2.8 \mathrm{~cm}$ as shown in Fig. 5 .

The characteristics of the rods are $\epsilon_{r}=2.4$ and $\sigma=0 \mathrm{~S} / \mathrm{m}$, the ones of water have been given above; the probing line length is 14 $\mathrm{cm}$.

The distance between the probing line and the rods is $y_{0}$ and the sampling interval on the probing line is $\lambda / 2=0.58 \mathrm{~cm}$. With four rotations of the rods around their symmetry point (four views), we obtain the amplitude of $\bar{K}(x, y)$ shown in Fig. 5.

\section{Rods with Different Electromagnetical Characteristics}

We investigate now the situation of two rods with different permittivities and conductivities (respectively, $\epsilon_{r_{1}}, \sigma_{1}$ and $\epsilon_{r_{2}}, \sigma_{2}$ ) to determine the minimum contrast between the two rods and with the surrounding medium. As above the distance between their centers is $2.8 \mathrm{~cm}$, the sampling interval on the probing line (length $14 \mathrm{~cm}$ ) is $\lambda / 2$.

In Fig. 6(a) is shown the cross section in $x$ direction at $y=0$ of the amplitude $|\tilde{K}(x, y)|$ with one view (without rotation) for two lossless rods $\left(\epsilon_{r_{1}}=2.4\right.$ and $\left.\epsilon_{r_{2}}=12\right)$.

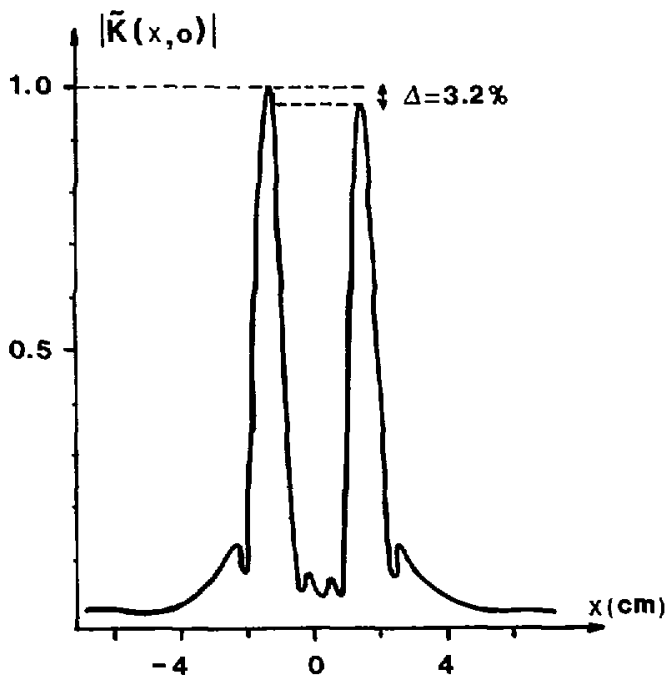

(a)

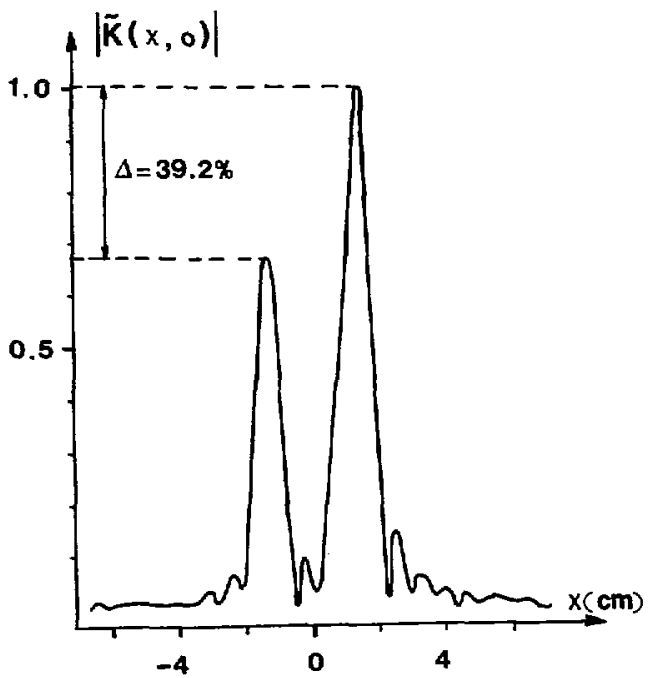

(b)

Fig. 6. (a) Modulus of $\tilde{K}(x, y)$ reconstruction (normalized with respect to the first rod) along the $x$ axis for two different dielectric rods $\left(\epsilon_{r_{1}}=2.4, \sigma_{1}=\right.$ $0.0 \mathrm{~S} / \mathrm{m}$ and $\left.\epsilon_{r_{2}}=12, \sigma_{2}=0.0 \mathrm{~s} / \mathrm{m}\right)$ separated by a distance of $2.8 \mathrm{~cm}$ and surrounded by water $\left(\epsilon_{r}=\not 74, \sigma=2.39 \mathrm{~S} / \mathrm{m}\right)$. (b) Modulus of $\tilde{K}(x, o)$ reconstruction (normalized with respect to the first rod) along $x$ axis $(y=0)$ for two lossy rods $\left(\epsilon_{r_{1}}=80.0, \sigma_{1}=2.39 \mathrm{~S} / \mathrm{m}\right.$ and $\epsilon_{r_{2}}=74.0, \sigma_{2}=1.0 \mathrm{~S} / \mathrm{m}$ in water $\left(\epsilon_{r}=74, \sigma=2.39 \mathrm{~S} / \mathrm{m}\right)$.

The difference level is about 3 percent for the modulus of $K(x, y)$. The reconstruction of two lossy rods filled with a medium close to the surrounding water medium is depicted in Fig. 6 (b). The characteristics of the two rods are, respectively, $\epsilon_{r_{1}}=$ $80, \sigma_{1}=2.39 \mathrm{~S} / \mathrm{m}$ and $\epsilon_{r_{2}}=74, \sigma_{2}=1.0 \mathrm{~S} / \mathrm{m}$ (for the water medium $\epsilon_{r}=74$ and $\sigma=2.39 \mathrm{~S} / \mathrm{m}$ ). The difference level is about 40 percent between the two rods for the amplitude $|\tilde{K}(x, y)|$ at $y=0$ ).

\section{INHOMOGENEOUS OBJECTS}

\section{A. Objects Composed of Fat, Muscle, and Bone}

A square object (side $a=8 \mathrm{~cm}$ ) with three dielectric layers simulating three kinds of human tissues (fat, muscle, and bone) surrounded by water and illuminated by a plane wave at $3 \mathrm{GHz}$ (Fig. 7) has been analyzed. The thickness of the fat medium is $1 \mathrm{~cm}$, and the dimensions of the bone are $2 \mathrm{~cm}$ over $2 \mathrm{~cm}$. The electromagnetic characteristics are, respectively, for fat, muscle, and bone, $\epsilon_{r_{f}}=5$ and $\sigma_{f}=0.2 \mathrm{~S} / \mathrm{m}, \epsilon_{r_{m}}=46$ and $\sigma_{m}=2 \mathrm{~S} / \mathrm{m}$, $\epsilon_{r_{b}}=8$ and $\sigma_{b}=0.22 \mathrm{~S} / \mathrm{m}$.

The probing line is located at $4.5 \mathrm{~cm}$ from the border of the 

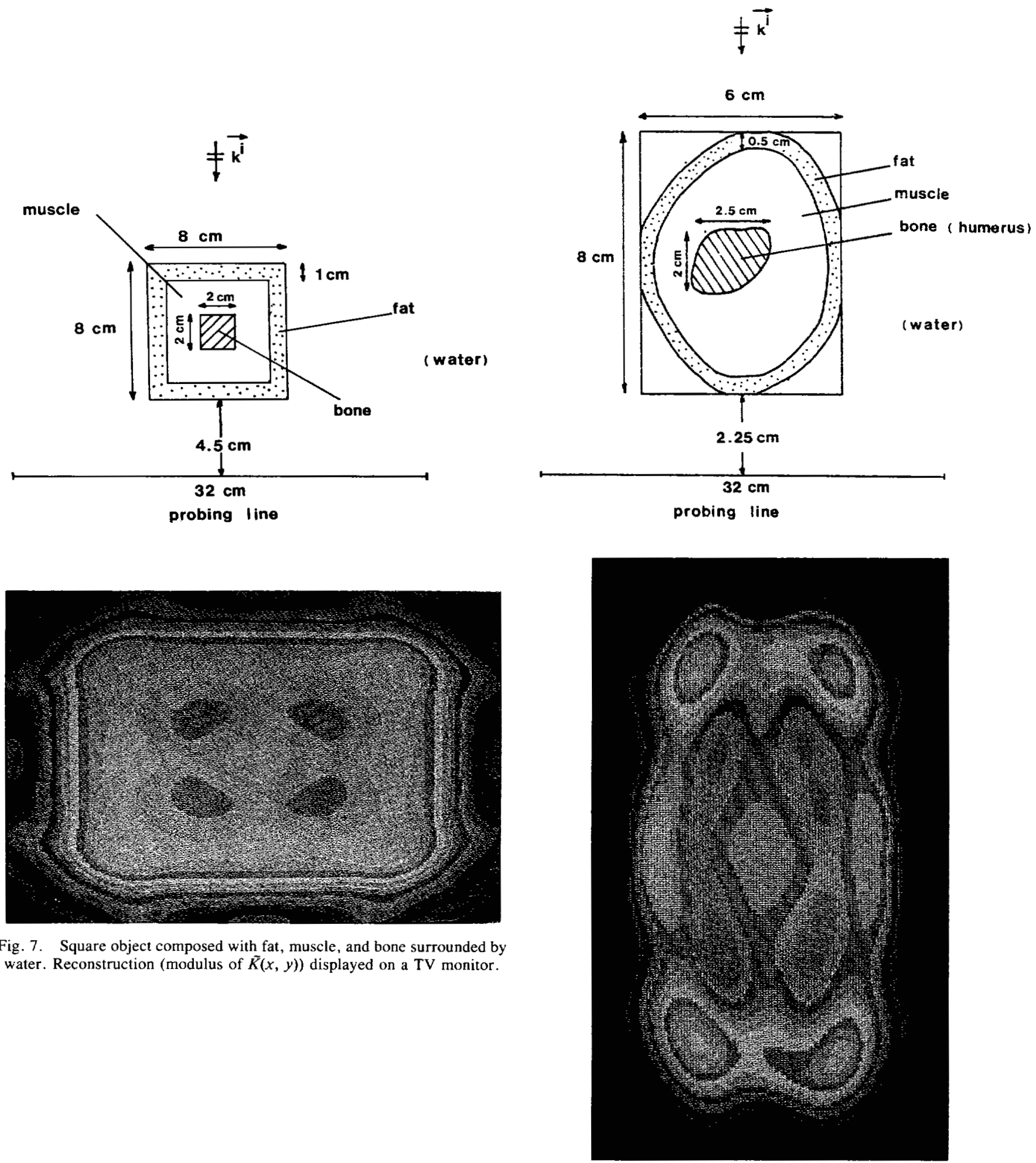

Fig. 7. Square object composed with fat, muscle, and bone surrounded by water. Reconstruction (modulus of $\tilde{K}(x, y)$ ) displayed on a TV monitor.

Fig. 8. Section of simulated human arm (upper segment) surrounded by water. Reconstruction (modulus of $\vec{K}(x, y)$ ). 


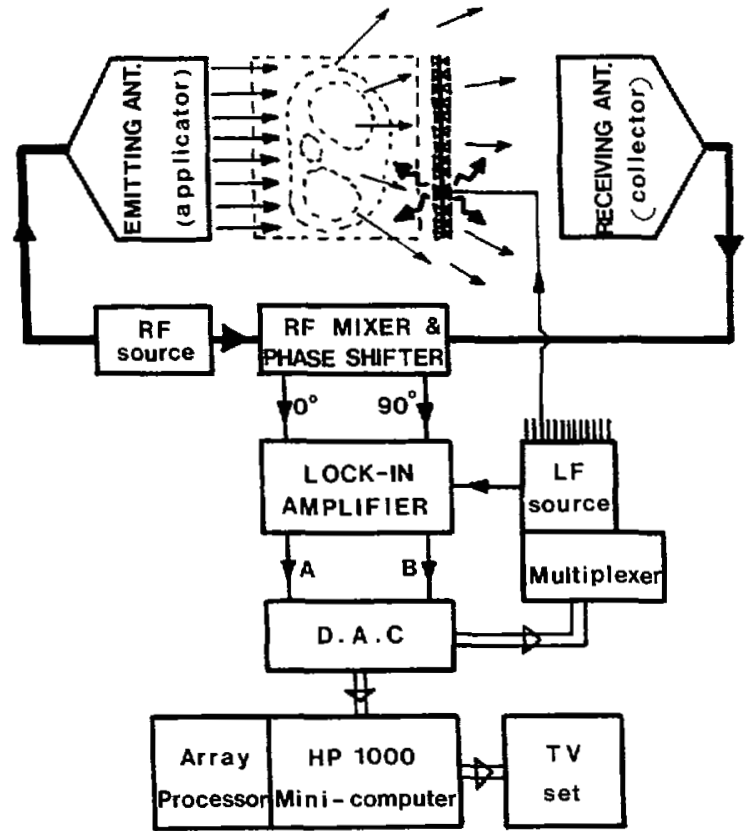

Fig. 9. Experimental block diagram at $3 \mathrm{GHz}$ using the modulated scattering technique.

object and its length is $32 \mathrm{~cm}$. The sampling interval is $\lambda / 2=$ $0.58 \mathrm{~cm}$. The object rotates in order to obtain four orthogonal views. From these views, the reconstruction of the normalized electrical current density $K(x, y)$ is achieved. The amplitude $|\tilde{K}(x, y)|$ is displayed on a TV monitor set (Fig. 7). Note that the object outline is relatively well described even if the number of views is very limited.

\section{B. Human Arm Simulation}

The reconstruction of a segment of a human arm (upper segment) from synthetically data is investigated (Fig. 8). The maximum length and width are, respectively, $8 \mathrm{~cm}$ and $6 \mathrm{~cm}$ and the average fat thickness is $0.5 \mathrm{~cm}$. For the direct problem (computation of the diffracted field outside or inside the object and in particular on the probing line), we use the moment method applied to (4). The length of the simulated probing line is $32 \mathrm{~cm}$ and it is separated from object by a distance of $2.25 \mathrm{~cm}$ (Fig. 8). The sampling interval on the data acquisition line is $\Delta x=0.58$ $\mathrm{cm}$. The normalized current distribution $K(x, y)$ is reconstructed using four orthogonal views and the modulus $|\tilde{K}(x, y)|$ is dis. played on a TV monitor screen. The fat thickness and bone presence are clearly visible.

\section{EXPERIMENTAL RESULTS}

Various configurations have been investigated in order to obtain a quasireal time measurement [13], [14], [15]. The method requires the knowledge of the normalized diffracted field on a segment line; and instead of moving a probe, which is time-consuming, the modulated scattering technique (MST) [13] , [15], [20] provides the speed of probe array without the need of microwave multiplexing. This technique allows a very short acquisition time: about $1 \mathrm{~ms}$ per point.

Fig. 9 shows the experimental setup operating in transmission.

The emitting source is an applicator constituted by a nonuniform guiding structure [21] which generates an incident field with a slowly varying amplitude and phase over a width $L$ (the maximum attenuation of $3 \mathrm{~dB}$ maximum is given for $L=16 \mathrm{~cm}$ ). The applicator is fed by a power generator so that the radiating

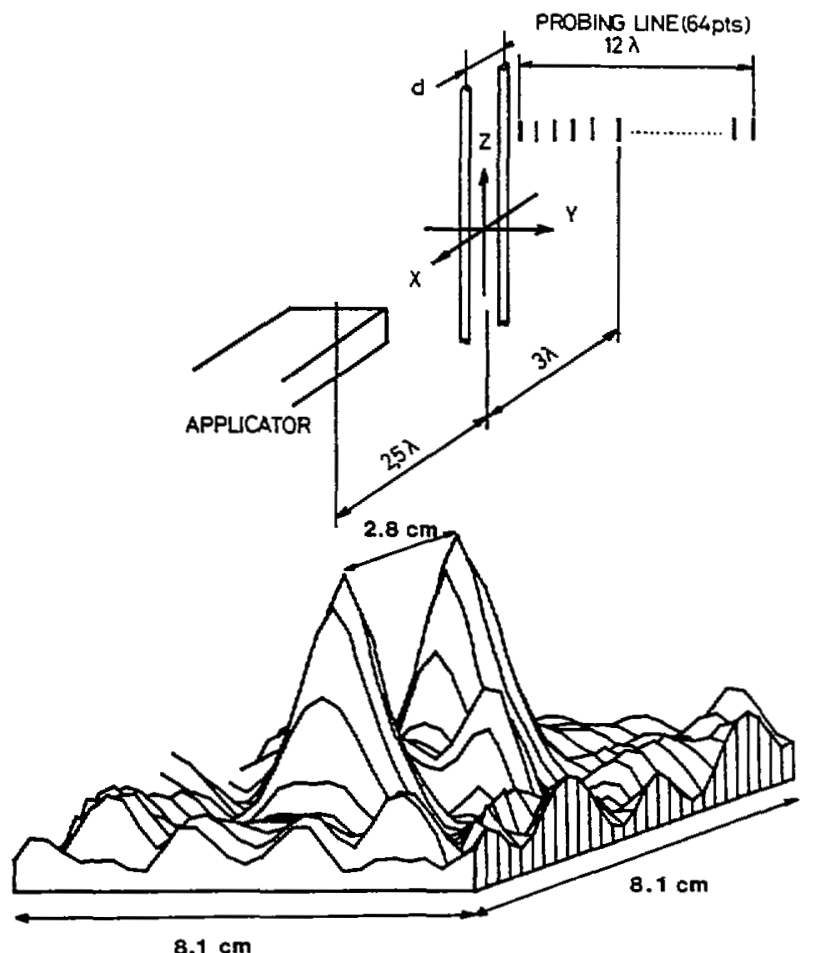

Fig. 10. Experimental setup. for resolution estimation with two dielectric rods $\left(\epsilon_{r_{1}}=\epsilon_{r_{2}}=2.4\right)$. Representation of $|\tilde{K}(x, y)|$.

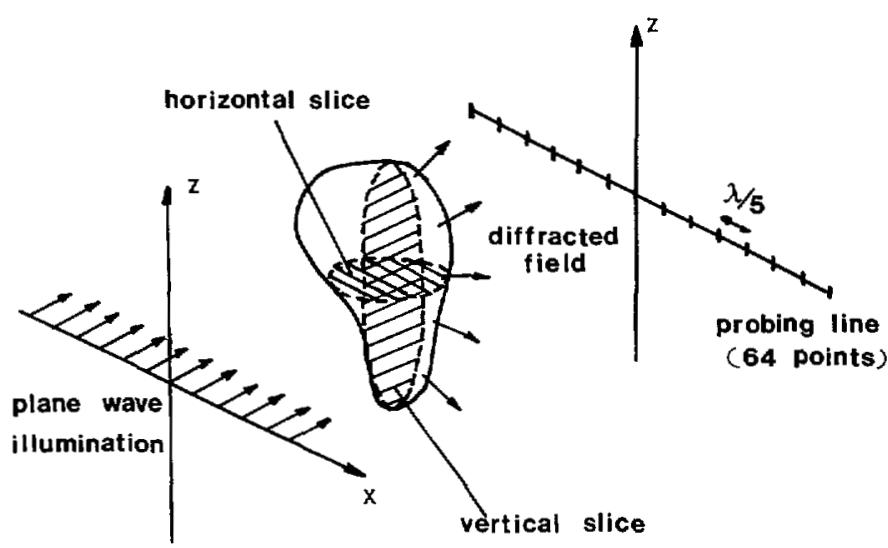

Fig. 11. Arrangement for three-dimensional object imaging (the object is translated along $z$ axis).

power density is less than $10 \mathrm{~mW} / \mathrm{cm}^{2}$ (safety standard); it is matched to water with a dielectric slab $\left(\epsilon_{r}=9\right)$.

The receiving system is constituted of an identical applicator in the aperture of which a linear array of 64 diodes-loaded dipoles is located. The interval between each dipole is $\lambda / 4.6$. Modulating successively each diode at low frequency rate $(1 \mathrm{kHz})$ results in a modulated scattered field proportional to the local field at the corresponding dipole location [15].

The object under test is immersed in a water tank in order to reduce the wavelength and to match the organ with the external medium.

Data acquisition and processing are performed with a HP 1000 minicomputer. The computation time is about $6 \mathrm{~min}$ (using four views). With the help of an array processor, a few seconds per image can be reached.

We consider an example of circular plexiglas rods diameter $\Phi=0.6 \mathrm{~cm}$ ) (permittivity $\epsilon_{r}=2.4$ ) with the same configuration as in the numerical simulation (Fig. 10).

The modulus $|\widetilde{K}(x, y)|$ of the normalized electric current, 


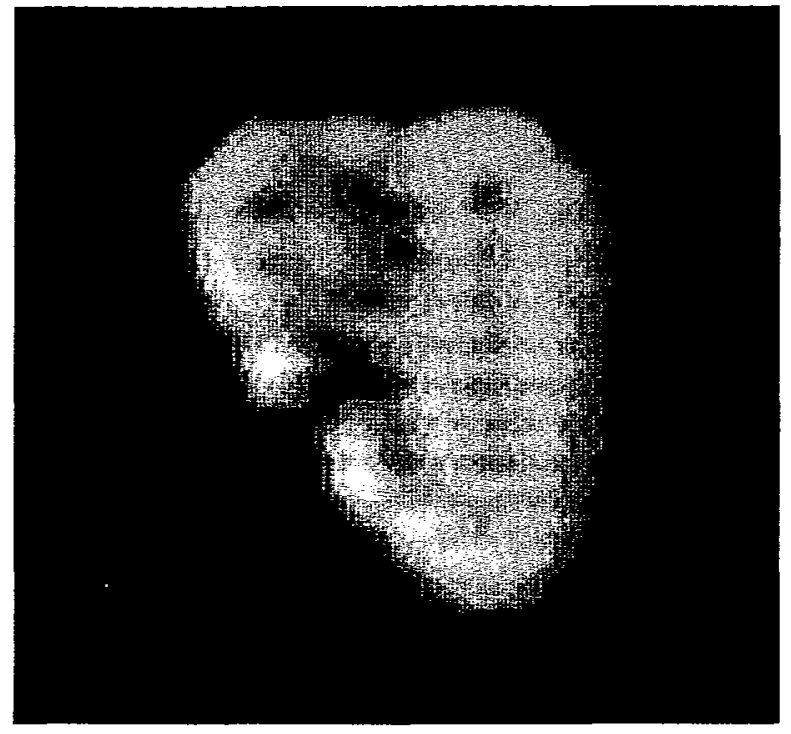

(a)

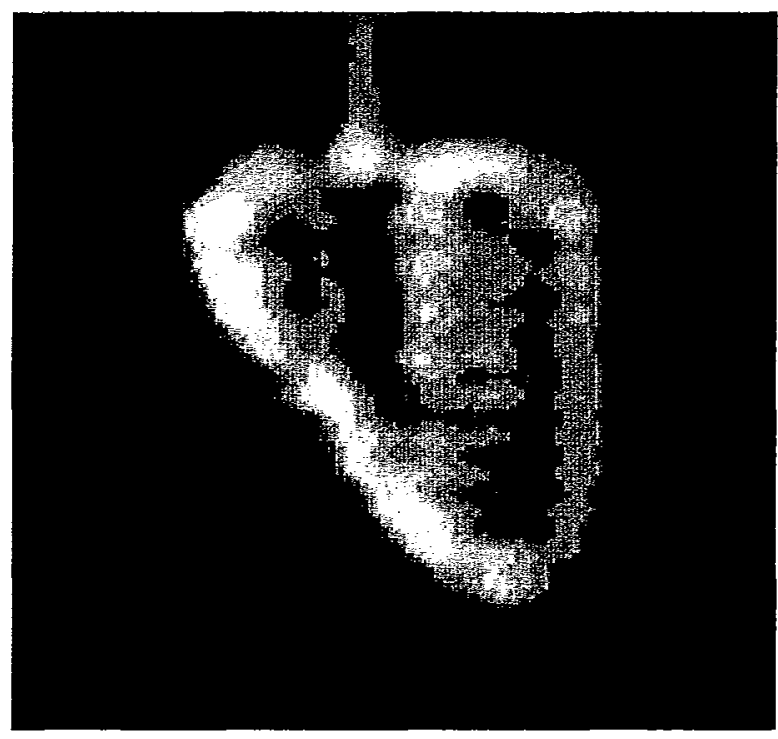

(b)

Fig. 12. (a) Vertical slice image of a nonperfused horse kidney. (b) Vertical slice image of a perfused horse kidney. The perfusion tube is at the top of the organ.

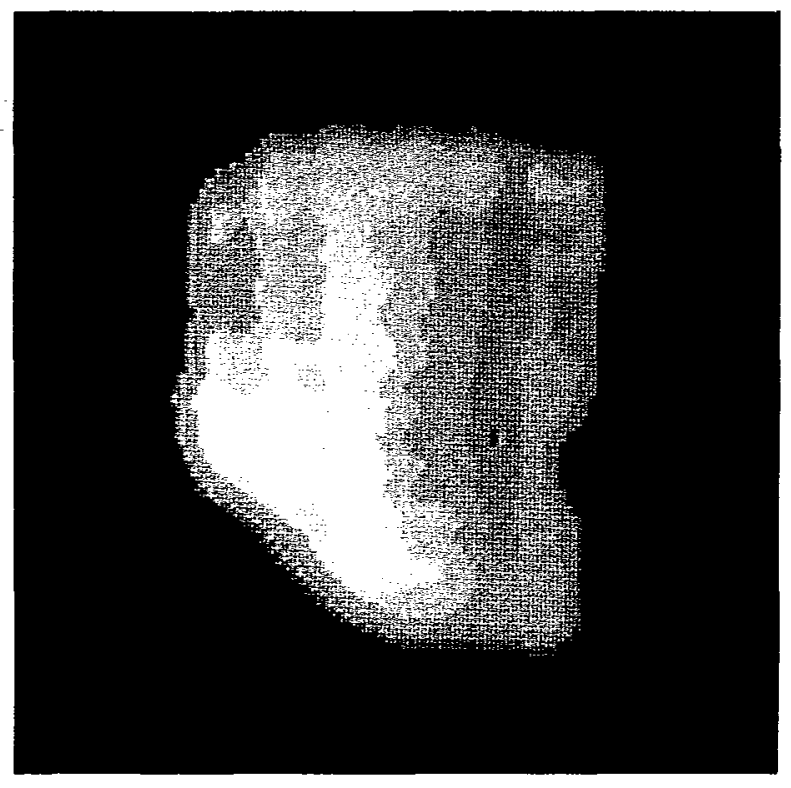

(a)

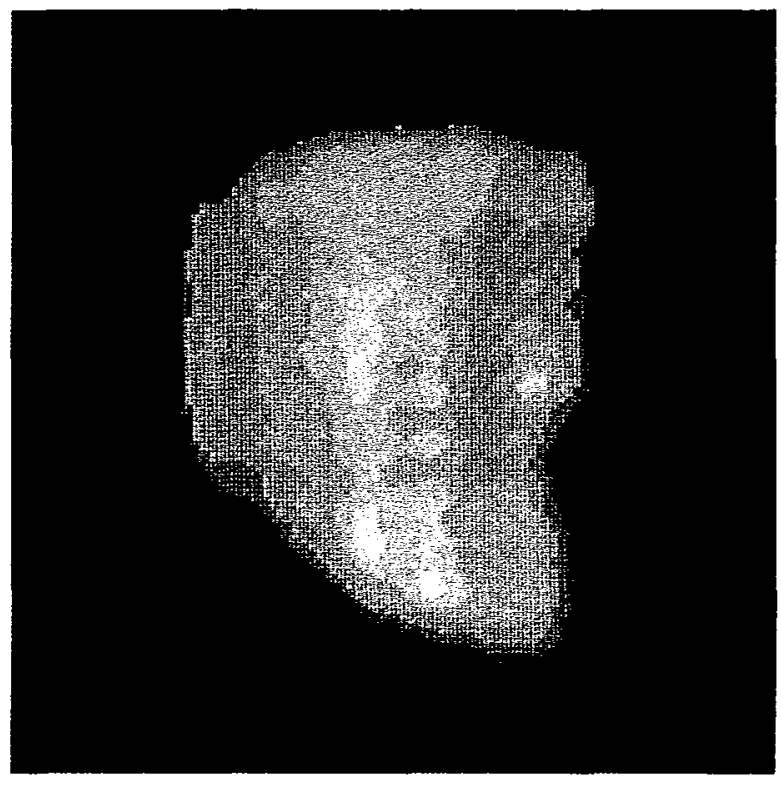

(b)

Fig. 13. (a) Vertical slice image of a perfused pony kidney. (The temperature perfusion is $38^{\circ} \mathrm{C}$.) (b) Vertical slice image of a perfused pony kidney. (The temperature perfusion is $41^{\circ} \mathrm{C}$ ). 
reconstructed from four orthogonal views, is shown in Fig. with 10 .

This method has been applied to perfused and nonperfused organs to investigate on the one hand the degree of water and on the other hand the influence of perfusion temperature on the organ.

As shown in Fig. 11, the object is translated on the $z$ axis in order to simulate a two-dimensional recording measurement with the probing line. Although the reconstruction method is based on cylindrical symmetry, it has been applied to three-dimensional object as an animal organ.

At each position of the probing line, we compute a horizontal tomographic image (horizontal slice as shown in Fig. 11). Various positions of the object along $z$ axis allow the computation of a vertical tomographic slice (Fig. 11). A right horse kidney whose dimensions are approximately $12 \mathrm{~cm} \times 15 \mathrm{~cm} \times 20 \mathrm{~cm}$ is im. mersed in warm water $\left(37^{\circ} \mathrm{C}\right)$.

Figs. 12(a) and 12(b) show the vertical image of a nonperfused and perfused horse kidney. In both images, outlines are well described (the image is displayed on a TV monitor with 16 color levels). The differences observed between the nonperfused and perfused cases illustrate the sensitivity of microwave imaging to the water content in biological tissues.

Other experiments have been made on a pony kidney to evaluate the influence of perfusion temperature. Figs. 12(a) and 12(b) show the conductivity changes due to a rise in temperature. As before, the organ is immersed in $37^{\circ} \mathrm{C}$ water and the perfusion temperature varies from $38^{\circ} \mathrm{C}$ (Fig. $13(\mathrm{a})$ ) to $41^{\circ} \mathrm{C}$ (Fig. 13 (b)). The temperature modifications can be visible with a resolution of $1^{\circ} \mathrm{C}$ approximately.

\section{CONCLUSION}

In this paper we present a numerical method and experimental technique for active microwave imaging of inhomogeneous bodies which allow tomographic reconstruction of the normalized electric current for biomedical applications or target recognition.

The method is based on the use of diffraction phenomena inside and outside the body using the diffracted field. By the use of performant algorithms as FFT, the tomographic reconstruction is carried out with low computing time (a few minutes on a minicomputer HP 1000 , a few seconds on an array processor).

Various examples are given from numerical simulations on homogeneous (dielectric rods) or inhomogeneous bodies (object with fat, muscle, bone), and from experimental results (dielectric rod, isolated animal organ) with illumination power compatible with the severe safety standards (atraumatic process).

The multiple illuminations (or rotations of the object) provide a spatial resolution of $\lambda / 2$ where $\lambda$ is the wavelength in the external medium (for a frequency of $3 \mathrm{GHz}$ and water as external medium, $\lambda=1.16 \mathrm{~cm}$ ).

Other applications as target analysis in air can be intended with this technique.

\section{APPENDIX}

\section{THREE-DIMENSIONAL CASE}

Consider a three-dimensional organ of volume $V$ illuminated by a plane wave linearly polarized along the $z$ axis. The diffracted field inside and outside the organ can tained from the following integral representation:

$$
\vec{E}^{d}(\vec{r})=\frac{1}{k^{2}}\left(\vec{\nabla} \vec{\nabla}+k^{2}\right) \iiint_{V} \vec{J}(\vec{r}) G\left(\vec{r}, \vec{r}^{\prime}\right) d \vec{r}^{\prime}
$$

$$
\begin{aligned}
\vec{J}(\vec{r})= & \left(k_{V}^{2}(\vec{r})-k^{2}\right) \vec{E}(\vec{r}) \\
G\left(\vec{r}, \vec{r}^{\prime}\right)= & \frac{e^{-i k\left|\vec{r}-\vec{r}^{\prime}\right|}}{4 \pi\left|\vec{r}-\vec{r}^{\prime}\right|} \\
= & -\frac{i}{2} \int_{-\infty}^{+\infty} \int_{-\infty}^{+\infty} \frac{e^{-i 2 \pi \nu_{1}\left(x-x^{\prime}\right)}}{\nu_{2}} e^{-i 2 \pi \nu_{2}\left|y-y^{\prime}\right|} \\
& \cdot e^{-i 2 \pi \nu_{3}\left(z-z^{\prime}\right)} d \nu_{1} d \nu_{3} \\
\nu_{2}= & \sqrt{k^{2}-4 \pi^{2}\left(\nu_{1}^{2}+\nu_{3}^{2}\right)} \\
\vec{E}(\vec{r})= & \vec{E}^{i}(\vec{r})+\vec{E}^{d}(\vec{r})
\end{aligned}
$$

Assume the depolarization is negligible; thus $\vec{E}^{d}$ is approximated by

$$
\vec{E}^{d}(\vec{r})=\iiint_{V} \vec{J}\left(\vec{r}^{\prime}\right) G\left(\vec{r}, \vec{r}^{\prime}\right) d \vec{r}^{\prime}
$$

with $\vec{E}^{d}$ as $\vec{E}^{i}$ parallel to $z$ axis. The plane wave is incident at an angle $\theta$ in the $x-z$ plane with respect to $y$-axis. Thus we can define $\Psi(\theta ; \vec{r})$ as

$$
\psi(\theta ; \vec{r})=\frac{E_{z}^{d}(\theta ; \vec{r})}{E_{z}^{i}(\theta ; \vec{r})} .
$$

$\psi(\theta ; \vec{r})$ satisfies the following equation according to (29).

$$
\psi(\theta ; \vec{r})=\left[E_{z}^{i}(\theta ; \vec{r})\right]^{-1} \iiint_{V} E_{z}^{i}\left(\theta ; \vec{r}^{\prime}\right) K\left(\theta ; \vec{r}^{\prime}\right) G\left(\vec{r}, \vec{r}^{\prime}\right) d \vec{r}^{\prime}
$$

with

$$
K(\theta ; \vec{r})=\left(k_{V}^{2}(\vec{r})-k^{2}\right)(1+\psi(\theta ; \vec{r})) .
$$

From (31) which is the three-dimensional extension of (4) and following the same steps as the two-dimensional case, one obtains the relation between the three-dimensional Fourier transform $\hat{K}(\theta, \vec{v})\left(\vec{\nu}=\left(\nu_{1}, \nu_{2}, \nu_{3}\right)\right)$ of $\hat{K}(\theta ; \vec{r})$ and the Fourier transform $\hat{\Psi}\left(\theta ; \nu_{1}, Y_{0}, \nu_{3}\right)$ of the data acquisition $\Psi\left(\theta ; X, Y_{0}, Z\right)$ on a recording plane at $Y=Y_{0}$ position.

$$
\begin{aligned}
& \hat{K}\left(\theta ; \alpha\left(\theta ; \nu_{1}, \nu_{3}\right), \beta\left(\theta ; \nu_{1}, \nu_{3}\right), \nu_{3}\right) \\
& \quad=2 i \sqrt{k^{2}-4 \pi^{2}\left(\nu_{1}^{2}+\nu_{3}^{2}\right)} e^{i \Omega\left(\nu_{1}, \nu_{3}\right) Y_{0}} \hat{\Psi}\left(\theta ; \nu_{1}, Y_{0}, \nu_{3}\right)
\end{aligned}
$$

with

$$
\begin{aligned}
\alpha\left(\theta ; \nu_{1}, \nu_{3}\right) & =\nu_{1} \cos \theta-\Omega\left(\nu_{1}, \nu_{3}\right) \sin \theta \\
\beta\left(\theta ; \nu_{1}, \nu_{3}\right) & =\nu_{1} \sin \theta+\Omega\left(\nu_{1}, \nu_{3}\right) \cos \theta \\
\Omega\left(\nu_{1}, \nu_{3}\right) & =\operatorname{Re}\left(\mu\left(\nu_{1}, \nu_{3}\right)\right) \\
\mu\left(\nu_{1}, \nu_{3}\right) & =-\frac{k}{2 \pi}+\frac{1}{2 \pi} \sqrt{k^{2}-4 \pi^{2}\left(\nu_{1}^{2}+\nu_{3}^{2}\right)} .
\end{aligned}
$$

\section{ACKNOWLEDGMENT}

The authors wish to thank Dr. M. Gautherie and Dr. J. L. Guerquin-Kern (Laboratoire de Thermologie Biomédicale, Faculté de Médecine of Strasbourg, France) for discussions and contribution to the experiments on animal organs. The cooperation with the Société d'Etude du Radant is greatly appreciated. The authors wish to thank Dr. Lesselier for his helpful comments on the manuscript. 


\section{REFERENCES}

[1] J. H. Jacobi, L. E. Larsen, and C. T. Hast, "Water immersed microwave antennas and their applications to microwave interrogation of biological targets," IEEE Trans. Microwave Theory Tech., vol. MTT27 , no. 1, pp. 70-78, 1979.

[2] L. E. Larsen and J, H. Jacobi, "Microwave scattering parameter imagery of an isolated canine kidney," Med. Phys., vol. 6, no. 5, pp. 394-403, 1979.

[3] J. H. Jacobi and L. E. Larsen, "Microwave time delay spectroscopic imagery of isolated canine kidney," Med. Phys., vol. 7, no. 1, pp. 1-7, 1980.

[4] P. S. Rao, K. Santosh, and E. C. Gregg, "Computer tomography with microwaves," Radiology, vol. 135, pp. 769-770, 1980.

[5] M. F. Adams and A. P. Anderson, "Three dimensional image construction technique and its application to coherent microwave diagnostics," Proc. Inst. Elec. Eng., vol. 127, pt. H, no. 3, pp. 138-142, 1980.

[6] R. Maini, M. F. Iskander, and C. H. Durney, "On the electromagnetic imaging using linear reconstruction techniques," Proc. IEEE, vol. 68, no. 12 , pp. $1550-1552,1980$.

[7] R. Maini, M. F. Iskander, C. H. Durney, and M. Berggren, "On the sensivity and the resolution of microwave imaging using ART," Proc. IEEE, vol. 69, no. 11, pp. 1517-1519, 1981.

[8] K. C. Tam and V. Perez-Mendez, "Tomographical imaging with limited angle input," J. Opt. Soc. Am., vol. 71, no. 5, pp. 582-592, 1981.

[9] H. Ermert, G. Fulle, and D. Hiller, "Microwave computerized tomography," in Proc. XIth European Microwave Conf., 1981. pp. 421-425.

[10] C. T. Herman, Image Reconstructions from Projections. New York: Springer-Verlag, 1979.

[11] M. Baribaud, F. Dubois, R. Floyrac, M. Kom, and S. Wang, "Tomographic image reconstruction of biological objects from coherent microwave diffraction data," Proc. Inst. Elec. Eng., vol. 129, Pt. H, pp. 356-359, 1982.

[12] C. Pichot, L. Jofre, G. Peronnet, C. Pichot, A. Izadnegahdar, J. C. Bolomey, "An angular spectrum method for inhomogeneous bodies reconstruction in microwave domain," in IEEE Antennas Propagat. Soc. Dig., vol. 2, 1982, pp. 664-667.

[13] J. C. Bolomey, L. Jofre, G. Peronnet, A. Jzadnegahdar, N. Berkane, M Solaimani, G. Collignon, Y. Michel, J. Saint, C. Szeles, M. Gautherie, and J. L. Guerquin-Kern, "A quasi real time microwave inverse scattering technique applicable to biomedical tomography," in Proc. URSI Symp. Nat. Radio Sci. Meet., 1982, p. 93.

[14] J. C. Bolomey, "La méthode de diffusion modulée: une approache au relevé des cartes de champs microondes en temps réel," L'Onde Electrique, vol. 62 , no. 5 , pp. 73-78, 1982.

[15] J. C. Bolomey, A. Izadnegahdar, L. Jofre, C. Pichot, G. Peronnet, and M. Solaimani, "Microwave diffraction tomography for biomedical applications," IEEE Trans. Microwave Theory Tech., vol. 30, no. 11, pp. 1998-2000, 1982.

[16] L. E. Larsen and J. H. Jacobi, "Microwaves offer promise as imaging modality," Diagnostic Imaging, pp. 42-47, 1982.

[17] M. F. Adams, and A. P. Anderson, "Synthetic aperture tomographic (S.A.T.) imaging for microwave diagnostics," Proc. Inst. Elec. Eng., vol. 129, pt. H, no. 2, pp. 83-88, 1982.

[18] R. K. Mueller, M. Kaveh, and G. Wade, "Reconstruction tomography and application to ultrasonics," Proc. IEEE, vol. 67, no. 4, pp. 567$587,1979$.

[19] J. H. Richmond, "Scattering by a dielectric cylinder of arbitrary crosssection shape," IEEE Trans. Antennas Propagat., vol. AP-13, no. 3, pp. 334-341, 1965.

[20] G. Collignon, Y. Michel, J. Saint, and J. C. Bolomey, "Quick microwave field mapping for large antennas," Microwave J., vol. 25 , no. 12 , pp. $129-132,1982$.

[21] N. Berkane and J. C. Bolomey, "Some applications of non-uniform waveguides," in Proc. URSI Symp. Nat. Radio Sci. Meet., 1981, p. 20.

[22] V. Barthes and G. Vasseur, An Inverse Problem for Electromagnetic Prospection in Applied Inverse Problems. New York: SpringerVerlag, 1978, pp. 325-329.

[23] G. Tyras, Radiation and Propagation of Electromagnetic Waves. New York: Academic, 1979.

[24] M. F. Iskander, R. Maini, C. H. Durney, and D. G. Bragg, A microwave method for measuring changes in lung water: Numerical solution," IEEE Trans. Biomed. Eng., vol, BME-28, no. 12, pp. 797$804,1981$.

[25] M. F. Iskander, C. H. Durney, D. G. Bragg, and B. H. Ovard, "A microwave method for estimating absolute value of average lung water," Radio Sci., vol. 17, no. 55, pp. 111S-117S, 1982.

[26] D. K. Ghodgaonkar, O. P. Gandhi, and M. J. Hagmann, "Estimation of complex permittivities of three-dimensional inhomogeneous biological bodies," IEEE Trans. Microwave Theory Tech., vol. MTT-31, no. 6, pp. 442-446, 1983.

[27] S. X. Pan and A. C. Kak, "A computational study of reconstruction algorithms for diffraction tomography: Interpolation versus backpropagation," IEEE Trans. Acoust. Speech and Signal Processing, vol. ASSP-31, no. 5, pp. 1262-1275, 1983.

[28] T. C. Guo, W. W. Guo, and L. E. Larsen, "A local field study of a water-immersed microwave antenna array for medical imagery and therapy," IEEE Trans. Microwave Theory Tech., vol. MTT-32, no. 8, pp. 844-860, 1984.

[29] M. Slaney, A. C. Kak, and L. E. Larsen, "Limitations of imaging with first-order diffraction tomography," IEEE Trans. Microwave Theory Tech., vol. MTT-32, no. 8, pp. 860-874, 1984.

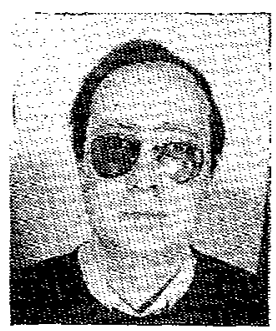

Christian Pichot was born in Rozay-en-Brie, France, on March 6, 1951. He received the M.S. degree from the University of Nice, France, in 1974, and the Doctorat Third Cycle and the Doctorat es Sciences degrees from the University of Paris-XI (Orsay) in 1977 and 1982, respectively.

He joined the Groupe d'Electromagnétisme of the Laboratoire des Signaux et Systémes in 1978. He is presently a Chargé de Recherche at the Centre National de la Recherche Scientifique (CNRS). His research activities are concerned with scattering, direct and inverse, and guided wave problems in inhomogeneous media. He received the Microwave Prize of the European Microwave Conference in Nürnberg in 1983.

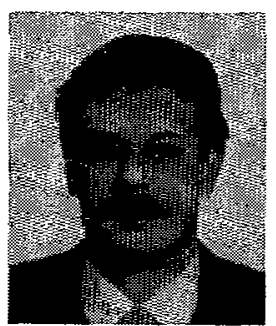

Luis Jofre was born in Mataró, Spain, in 1956. He received the Ingeniero and Doctor Ingeniero degrees in Telecommunication Engineering, both from the Unisersidad Politécnica of Barcelona (UPB), in 1978 and 1982, respectively.

In 1978 he was employed as Research Assistant in the Electrophysics Department at U.P.B. where he worked on analysis and near-field measurement of antennas. During 1981 he joined the Ecole Supérieure d'Electricité, where he was involved in microwave imaging techniques for biomedical applications. Since 1982 he is an Associate Professor at he U.P.B. where he is engaged in research in the areas of electromagnetic wave diffraction and antennas, with specific emphasis on microwave holography and near-field techniques. He received the Microwave Prize of the European Microwave Conference in Nürnberg in 1983.

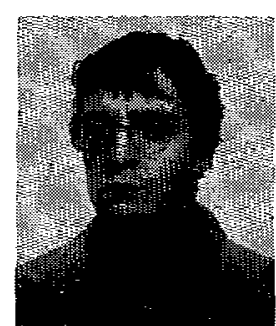

Guillaume Peronnet was born in Grenoble, France, in 1956. He received the Engineer degree from Ecole Supérieure d'Electricité in 1979 with radio communications specialization.

Since 1980 he has been a Chef de Travaux Adjoint in ESE where he is engaged in research in electromagnetism and particularly in microwave imaging. $\mathrm{He}$ received the Microwave Prize of the European Microwave Conference in Nürnberg in 1983.

Jean-Charles Bolomey, for a photograph and biography please see page 285 of the March 1985 issue of this TRANSACTIONS. 

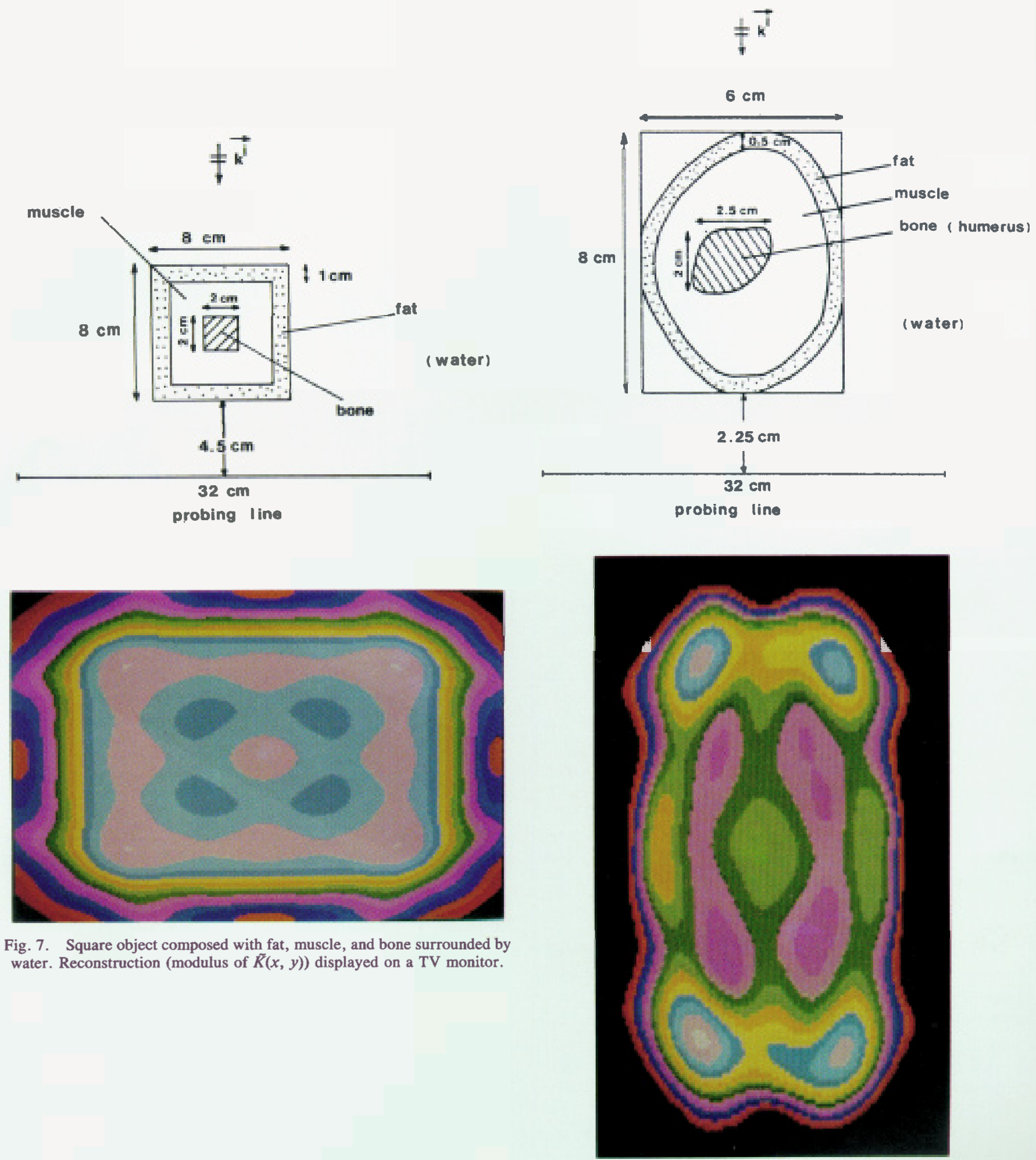

Fig. 7. Square object composed with fat, muscle, and bone surrounded by water. Reconstruction (modulus of $\tilde{K}(x, y)$ ) displayed on a TV monitor.

Fig. 8. Sectan of sinnulated human arm (upper segment) surrounded by water. Reconstruction (modulus of $\tilde{K}(x, y)$ ). 


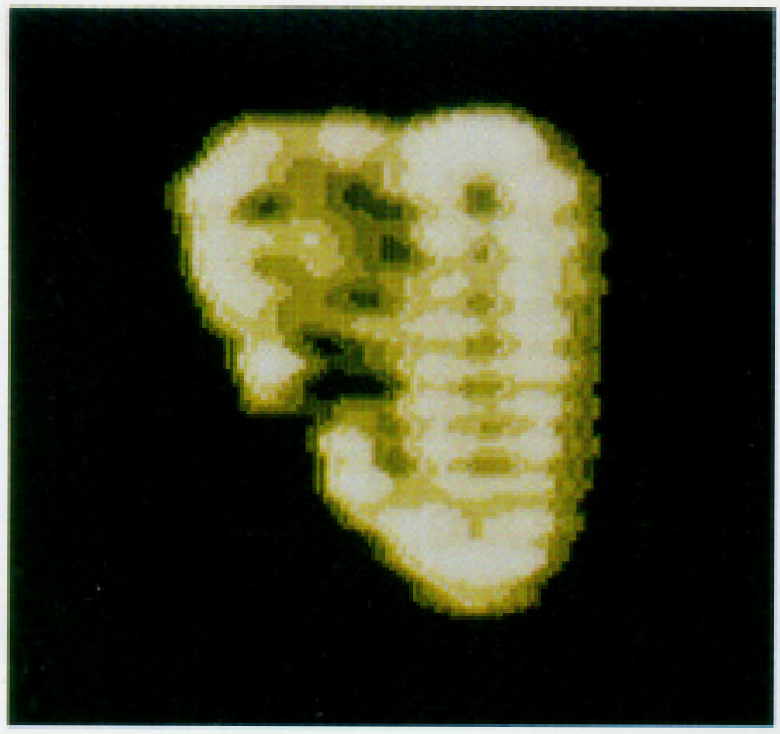

(a)

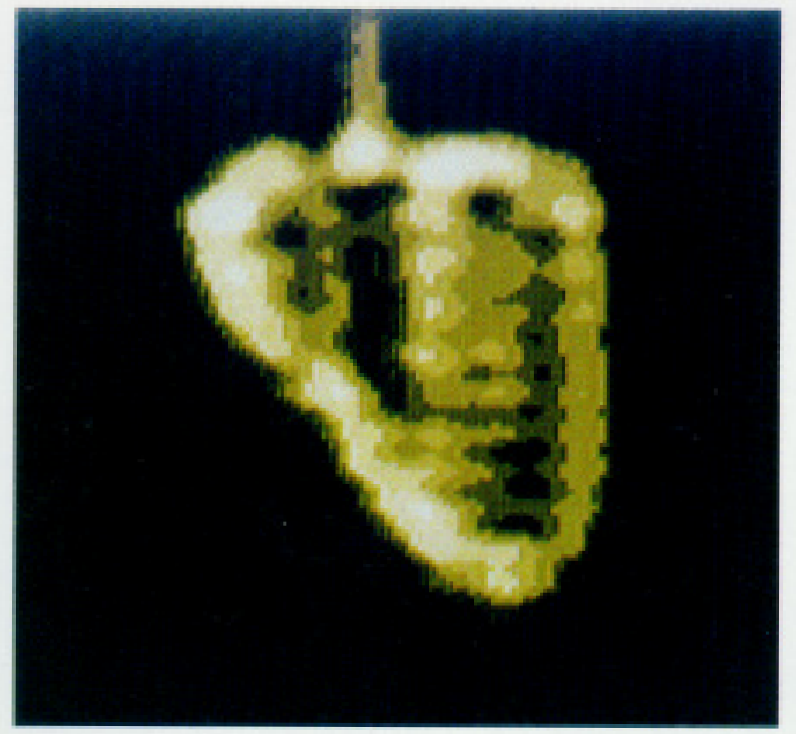

(b)

Fig. 12. (a) Vertical slice image of a nonperfused horse kidney. (b) Vertical slice image of a perfused horse kidney. The perfusion tube is at the top of the organ.

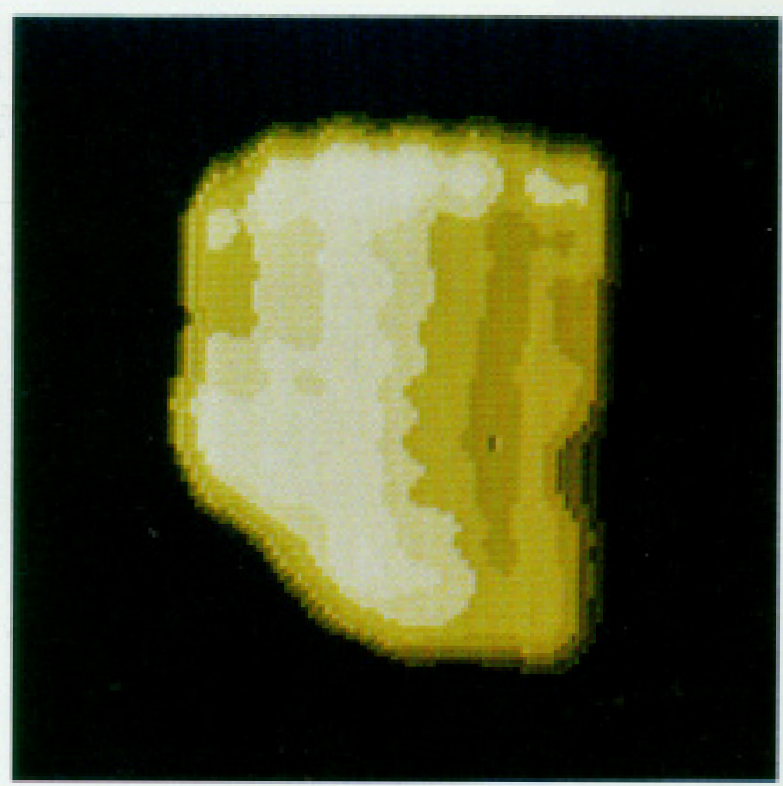

(a)

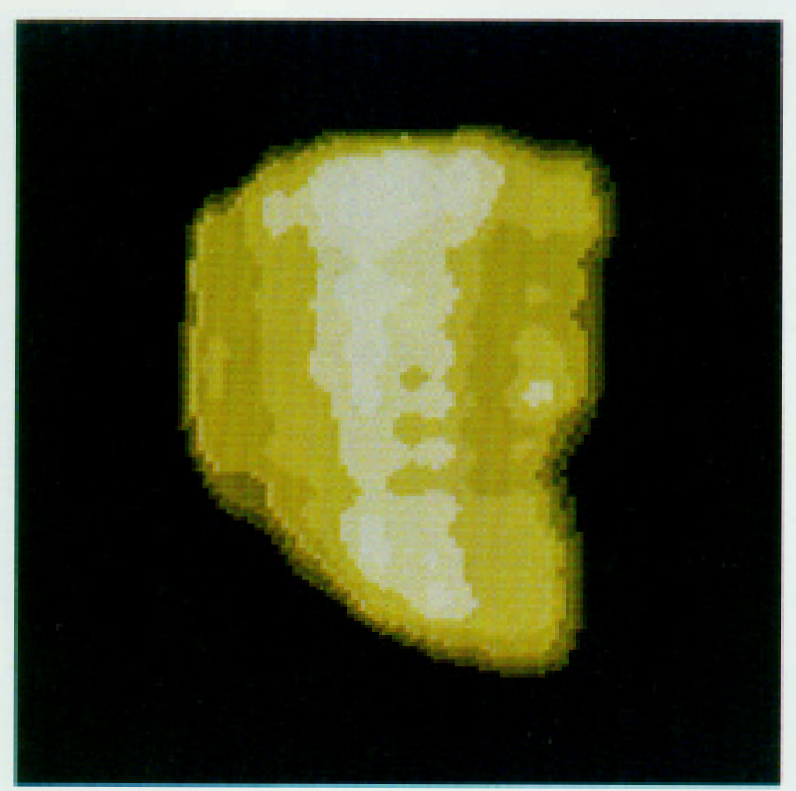

(b)

Fig. 13. (a) Vertical slice image of a perfused pony kidney. (The temperature perfusion is $38^{\circ} \mathrm{C}$.) (b) Vertical slice image of a perfused pony kidney. (The temperature perfusion is $41^{\circ} \mathrm{C}$ ). 